OPEN ACCESS

Edited by:

Thomas FitzGerald, University of Massachusetts Boston,

United States

Reviewed by:

Brett Diamond,

Tufts Medical Center, United States

Eric Chi-ching Ko,

University of Massachusetts Medical

School, United States

*Correspondence: Albert Chi-Yan Chan acchan@hku.hk

Specialty section: This article was submitted to

Radiation Oncology,

a section of the journal

Frontiers in Oncology

Received: 21 October 2021 Accepted: 15 November 2021 Published: 07 December 2021

Citation:

Chiang C-L, Chiu KW-H, Lee FA-S, Kong F-MS and Chan AC-Y (2021)

Combined Stereotactic Body

Radiotherapy and Immunotherapy

Versus Transarterial

Chemoembolization in Locally Advanced Hepatocellular Carcinoma: A Propensity Score Matching Analysis.

Front. Oncol. 11:798832. doi: 10.3389/fonc.2021.798832

\section{Combined Stereotactic Body Radiotherapy and Immunotherapy Versus Transarterial Chemoembolization in Locally Advanced Hepatocellular Carcinoma: A Propensity Score Matching Analysis}

\author{
Chi-Leung Chiang ${ }^{1}$, Keith Wan-Hang Chiu ${ }^{2}$, Francis Ann-Shing Lee ${ }^{3}$, \\ Feng-Ming Spring Kong ${ }^{1}$ and Albert Chi-Yan Chan ${ }^{4 *}$
}

\begin{abstract}
1 Department of Clinical Oncology, The University of Hong Kong, Hong Kong, Hong Kong SAR, China, ${ }^{2}$ Department of Diagnostic Radiology, The University of Hong Kong, Hong Kong, Hong Kong SAR, China, ${ }^{3}$ Department of Clinical Oncology, Tuen Mun Hospital, Hong Kong, Hong Kong SAR, China, ${ }^{4}$ Department of Surgery, The University of Hong Kong, Hong Kong, Hong Kong SAR, China
\end{abstract}

Immunotherapy has achieved modest clinical activity in HCC patients. Propensity score matching analysis was conducted to compare the efficacy and safety of combined stereotactic SBRT-IO versus TACE in patients with locally advanced HCC in a tertiary center of Hong Kong. Patients with locally advanced HCC who were medically inoperable for, refractory to, or refused to curative surgical interventions were eligible. The primary outcome was PFS; the secondary outcomes were OS, ORR as per mRECIST version 1.1, and TRAEs. Matching pair analysis was performed to compare the clinical outcomes. A total of 226 patients were eligible. Approximately 16 patients in the SBRT-IO group were matched with 48 patients treated with TACE. The median tumor size was $10 \mathrm{~cm}$ (range: $2.9-19.6 \mathrm{~cm}$ ) and $20.3 \%$ of the patients had portal vein invasion. The 12- and 24-month PFS were significantly better in the SBRT-IO group (93.3\% vs $16.7 \%$ and $77.8 \%$ vs $2.1 \%$, respectively, $\mathrm{p}<0.001$ ); the 12 - and 24-month OS were also better in the SBRT-IO arm (93.8\% vs $31.3 \%$ and $80.4 \%$ vs $8.3 \%$, respectively, $p<0.001$ ). The ORR was $87.5 \%$ (CR: 50\%, PR: 37.5\%) in SBRT-IO arm compared to $16.7 \%$ (CR: 2.4\%, PR: $14.3 \%$ ) in those receiving TACE alone ( $p<0.001)$. There were fewer $\geq$ grade 3 TRAE (60.4\% vs $18.8 \%, p=$ 0.004 ) and treatment discontinuations ( $25 \%$ vs $12.5 \%, p=0.295$ ) due to adverse events in the SBRT-IO arm. SBRT-IO had significant superior survival and less treatment toxicity than TACE in patients with locally advanced HCC. Our results provide rationale for studying this combination therapy in prospective randomized trials.

Keywords: transarterial chemoembolization, stereotactic body radiotherapy, immunotherapy, hepatocellular carcinoma, immune checkpoint inhibitors, radiotherapy, liver cancer 


\section{INTRODUCTION}

Recent advances in cancer immunotherapy have profoundly influenced the care of patients with hepatocellular carcinoma (HCC). Programmed cell death protein 1/programmed deathligand 1 (PD-1/PD-L1) targeted therapies have been increasingly used as first-line and second-line treatments of patients with advanced HCC (1-3). However, response rates are modest; overall the response rates to anti PD-1 monotherapy is around $20 \%$, and even combination therapies of atezolizumab/ bevacizumab, pembrolizumab/lenvatinib, or nivolumab/ ipilimumab are not higher than 50\% (4-6). Because the primary resistance of HCC may underlie these low response rates, strategies to overcome these primary or secondary resistances to immune checkpoint inhibitors (ICI) using combination therapies such as combined stereotactic body radiotherapy and immunotherapy (SBRT-IO) are under investigation.

Radiotherapy (RT) has been shown to enhance immunotherapeutic effects. RT can prime the immune system by enhancing antigen presentation, promoting the infiltration of cytotoxic T-cells, and reprogramming the tumor microenvironment against the immune evasion of cancer, while ICI can reverse the RT-mediated exhaustion pathway (7). SBRT-IO has been reported in several cancers, potentially improve the clinical outcome of patients $(8,9)$. But, evidence on the combination of ICI and SBRT in HCC patients is lacking.

We recently reported encouraging results of SBRT-IO in a small pilot of patients with locally advanced unresectable HCC (10). This study aimed to compare the clinical outcomes of these patients treated with SBRT-IO versus TACE, the current the standard of care in this population.

\section{MATERIALS AND METHODS}

\section{Study Design and Population}

This was an Institutional Review Board (IRB) approved retrospective study (IRB number: UW-20-674) conducted at a tertiary referral center of Hong Kong. Data was retrieved from a prospectively collected HCC database at the Queen Mary Hospital, Hong Kong. Patients with histological or radiological HCC, who were ineligible for, refractory to, or refused curative surgical interventions, were candidates of loco-regional treatment. All cases were discussed in the multi-disciplinary tumor board. TACE was the standard of care. An experimental treatment of SBRT-IO was offered as an alternative since 2017

\footnotetext{
Abbreviations: CR, Complete remission; DCR, Disease control rate; ECOG, Eastern Cooperative Oncology Group; HCC, Hepatocellular carcinoma; HR, Hazard ratio; ICI, Immune checkpoint Inhibitor; IRB, Institutional review board; mRECIST, Modified Response Evaluation Criteria in Solid Tumors; ORR, Objective response rate; OS, Overall survival; PD-1, Program cell death protein 1; PD-L1, Programmed death-ligand 1; PFS, Progression-free survival; PR, Partial remission; RTOG, Radiation Therapy Oncology Group; SBRT-IO, Combined stereotactic body radiotherapy and immunotherapy; TACE, Transarterial chemoembolization; TRAE, Treatment-related adverse event.
}

based on the potential synergistic effect between SBRT and ICI (10). While the optimal sequence of combining SBRT and ICI remains controversial, we deliver SBRT prior immunotherapy based on the immune-activation property of radiation to sensitize the tumor for subsequent PD-1 inhibitors (11). The advantages and disadvantages of these local treatments were informed to patients and the final treatment depends on patients' decisions.

The inclusion criteria were as follows: (a) patients were ineligible or refractory to curative surgical interventions; (b) a Child-Pugh (CP) liver score of A5 to B7; (c) tumor nodules $\leq 5$; (d) no main trunk of portal vein invasion (Vp4); (e) no prior systemic therapy, and (f) absence of extra-hepatic metastasis, ascites or encephalopathy. There were no limits on the maximum diameters of tumors.

All patients who received SBRT-IO or TACE from January 2010 to May 2020 were included. Propensity score matching was performed using the nearest neighboring method in 3:1 ratio according to age, sex, tumor size, numbers, and portal vein invasion between the TACE and SBRT-IO groups.

\section{Transarterial Chemoembolization}

TACE was performed by supra-selective cannulation of all the branches supplying the tumor. The emulsion was prepared by mixing lipiodol with cisplatin $(1 \mathrm{mg} / \mathrm{ml})$ in a 1:1 ratio using the pumping method, which was then slowly injected under fluoroscopic monitoring according to the size of the tumor and the arterial blood flow. TACE was repeated in eight-week intervals (12).

\section{Stereotactic Body Radiotherapy and Immunotherapy}

For SBRT planning, patients were immobilized via a vacuum foam bag (Vac-LokTM; MEDTEC, Iowa, USA) and active breathing control to reduce the amplitude of liver motion. Imaging was performed on the inhale breath-hold contrast computed tomography (CT). GTV was defined as tumor focus that was visualized on contrast imaging. The clinical target volume (CTV) was defined as GTV plus a margin of $0-3 \mathrm{~mm}$. The individualized PTV margins were formulated to compensate the respiratory motion and set-up errors. Cone beam CT was acquired on board before each treatment. The largest tumor was selected as index lesion of SBRT, while maximum three nodules were allowed provided that the liver tolerance dose can be met. The dose was prescribed according to the Radiation Therapy Oncology Group (RTOG) 1112 protocol (23). The total dose of 25 to $50 \mathrm{~Gy}$ in five fractions was allowed per institutional protocol. The prescription isodose should encompass $95 \%$ of PTV. The final dose was determined such that a maximum tumoricidal dose could be delivered to tumors while respecting the tolerance dose of OAR to the limits of RTOG 1112.

Among our patient cohort, a total dose ranging from 25 to $37.5 \mathrm{~Gy}$ in five fractions was given during 1-2 weeks. At 2 weeks after SBRT, intravenous nivolumab at a dose of $3 \mathrm{mg} / \mathrm{kg}$ was started and was given every 2 weeks, median 10 cycles (range: 1 20 doses) were given. 


\section{Evaluation of Treatment Response and Decision on Treatment Discontinuation}

Contrast computed tomography was performed every 8-12 weeks in the first two years. All radiological responses were evaluated according to the Modified Response Evaluation Criteria for Solid Tumors (mRECIST) version 1.1. Treatmentrelated adverse events (TRAEs) were graded using the National Cancer Institute Common Terminology for Adverse Events (CTCAE) version 4.0. Treatments were continued until disease progression, unacceptable toxicities, refusal of patients, or achieved radiological complete remission (CR).

\section{Statistical Analysis}

The primary endpoint was progression-free survival (PFS), which was defined as the period from the date of commencement of the treatment to the time of disease progression (either progression of treated lesion, elsewhere in liver, or development of distant metastases), as per mRECIST, or death, whichever occurred earlier. The secondary endpoints included overall survival (OS), objective response rate (ORR), TRAEs, and liver function deterioration. OS was defined as the period from the date of commencement of the study treatment to the date of death or last follow-up, whichever occurred earlier. Radiological response was recorded per lesion according to the mRECIST. Disease control rate (DCR) was defined as percentage of patient attained radiological complete response $(\mathrm{CR})$, partial response (PR) or stable disease for $\geq 6$ months. Liver function deterioration was defined as progression of Child-Pugh score of $\geq 2$.

Continuous variables were presented as medians and ranges. Comparison between the groups was carried out using the Chisquared or Mann-Whitney U test where appropriate. Survivals were studied with the Kaplan-Meier method. Cox proportional hazard regression model was used to determine independent prognostic factors. Statistical significance was defined as $p<0.05$, and all the performed tests were two-tailed. Data was analyzed using R version 3.25 (Vienna, Austria).

\section{RESULTS}

\section{Patients and Treatments}

A total of 226 patients with HCC were eligible and enrolled in the present study, namely, 210 patients who initially received TACE and the remaining 16 who were treated with SBRT-IO. Table 1 shows the baseline and tumor characteristics of all the patients and their significances for clinical outcome.

The SBRT-IO group had higher percentage of patients with Barcelona Clinic Liver Cancer (BCLC) stage C disease and portal vein invasion; median size of tumor was larger in the SBRT-IO group (10 cm vs. $6.95 \mathrm{~cm}, \mathrm{p}=0.016)$. After propensity score

TABLE 1 | Baseline demographics and tumour characteristics of all patients.

\begin{tabular}{|c|c|c|c|c|c|}
\hline & \multicolumn{3}{|c|}{ Before propensity score matching } & \multicolumn{2}{|c|}{ After propensity score matching } \\
\hline Sex (n, \% male) & $158(75.2)$ & $14(87.5)$ & 0.268 & $43(89.6)$ & 0.817 \\
\hline Hepatitis B carrier (n, \%) & $129(61.4)$ & $12(75.0)$ & 0.280 & $26(54.2)$ & 0.142 \\
\hline ECOG 0-1 (n, \%) & $192(91.4)$ & $12(75.0)$ & 0.439 & $45(93.8)$ & 0.407 \\
\hline Child-Pugh class A (n, \%) & $182(86.7)$ & $14(87.5)$ & 0.925 & $46(95.8)$ & 0.233 \\
\hline 2 & $121(57.6)$ & $7(43.8)$ & & $32(66.7)$ & \\
\hline 3 & $13(6.2)$ & $1(6.2)$ & & $1(2.1)$ & \\
\hline Albumin (g/L) & $37(17-48)$ & $39(30-45)$ & 0.250 & $37(25-45)$ & 0.192 \\
\hline Bilirubin ( $\mu \mathrm{mol} / \mathrm{L})$ & $13(4-55)$ & $15(8-122)$ & 0.171 & $12.5(4-39)$ & 0.149 \\
\hline Platelet $\left(\times 10^{9} / \mathrm{L}\right)$ & $169.5(25-551)$ & $234(79-402)$ & 0.069 & $226(66-522)$ & 0.773 \\
\hline INR & $1.1(0.8-2.3)$ & $1.1(1-1.5)$ & 0.339 & $1.1(0.9-1.6)$ & 0.543 \\
\hline Tumor number (n, \%) & & & 0.518 & & 0.460 \\
\hline 1 & 89 (42.4) & $9(56.2)$ & & 27 (56.3) & \\
\hline 2 & $26(12.4)$ & $2(12.5)$ & & $1(2.1)$ & \\
\hline$\geq 3$ & $95(45.2)$ & $5(31.3)$ & & $20(41.6)$ & \\
\hline Tumor size $(\mathrm{cm})^{\star}$ & $6.95(1-19.6)$ & $10(3.4-18)$ & 0.016 & $10.4(2.68-19.6)$ & 1.000 \\
\hline Portal vein invasion $(n, \%)$ & $19(9.1)$ & $3(18.8)$ & 0.001 & $10(20.8)$ & 0.827 \\
\hline AFP $\geq 200 \mathrm{ng} / \mathrm{ml}(\mathrm{n}, \%)$ & $84(40.0)$ & $7(43.8)$ & 0.768 & $21(43.8)$ & 1.000 \\
\hline Range & $1-1,458,960$ & $3-499,988$ & & $2-362,901$ & \\
\hline
\end{tabular}

TACE, transarterial chemoembolization; SBRT-IO, combined stereotactic body radiotherapy and immunotherapy; ECOG, Eastern Cooperative Oncology Group; INR, international normalized ratio; BCLC, Barcelona Clinic Liver Cancer; AFP, alpha-feto protein.

${ }^{*}$ Tumor size of the largest lesion. 
matching, a total of 48 patients treated with TACE were identified to match the 16 patients treated with SBRT-IO. No significant difference was observed between-group. Overall, around $90 \%$ of analyzed patients were male and had a performance status of ECOG $0-1$, and $60 \%$ were hepatitis B carrier.

\section{Patients Population Between TACE and SBRT-IO}

Among the 64 included patients after matching, the median size of tumor was $10 \mathrm{~cm}$ (range: $3.4-19.6 \mathrm{~cm}$ ) and $20.3 \%$ of the patients had portal vein invasion. Among the 48 patients matched in the TACE arm, median 2 sessions of TACE (range: 1-16) were given. For the SBRT-IO arm, a median dose of $35 \mathrm{~Gy}$ (range: 27.5-37.5 Gy) was prescribed and median 10 cycles of nivolumab (range: 1-20 doses) were given. Total 24 lesions were irradiated in SBRT-IO arm. $(\mathrm{N}=11$, single lesion; $\mathrm{N}=2$, two lesions; $\mathrm{N}=3$, three lesions). One and six patients received postprogression therapies in the SBRT-IO and TACE arms, respectively. Neither patient in the TACE arm received SBRT or immunotherapy, nor patient in SBRT-IO arm received TACE after progression (refer to Table $\mathbf{S 1}$ for detailed information).

\section{Overall Survival and Progression-Free Survival Between TACE and SBRT-IO}

The survival data was censored on December 31, 2020. The median follow-up time of the SBRT-IO and matched TACE groups were 12.7 months (range: 2.5-36.1 months) and 7.4 months (range: 0.2-57.2 months), respectively. The 6-, 12-, and 24-month PFS were better in the SBRT-IO group $(93.3 \%$ vs. $37.5 \%, 93.3 \%$ vs. $16.7 \%$, and $77.8 \%$ vs. $2.1 \%$, respectively, $\mathrm{p}<0.001)$. The median PFS of the SBRT-IO group was not reached (range: 1.9-36.1 months) compared to 4.83 months (range: 0.2-42.2 months) of the TACE group. The 6-, 12-, and 24-month OS were also better in the SBRT-IO group (93.8\% vs. $54.2 \%, 93.8 \%$ vs. $31.3 \%$, and $80.4 \%$ vs. $8.3 \%$, respectively, p $<0.001)$. The median OS of the SBRT-IO group was not reached (range: 2.5-36.1 months) compared to 7.44 months (range: $0.2-$ 57.2 months) of the TACE group (as shown in Figure 1).

At the time of analysis, there were three deaths out of 16 patients in the SBRT-IO arm, and all the 48 patients died in the matched TACE group. All of the three patients who died in the SBRT-IO group showed no evidence of disease progression; two died of community-acquired pneumonia and one died of hemobilia. In the matched TACE group, 37 deaths $(77.1 \%)$ were cancer related, four died of pulmonary causes, two died of intra-cranial hemorrhage, and one died of myocardial infarction, liver abscess, liver decompensation, intestinal obstruction, or unknown cause (each). Among patients in the matched TACE group, intra-hepatic progression (90.6\%) represented the dominant mode of failure; there were no significant differences in the PFS or OS between the different treatment periods from 2010 to 2020 (as shown in Figure S1).

Under the multi-variable analysis, SBRT-IO, was an independent predictor of better OS (hazard ratio $(\mathrm{HR})=0.14$, range: $0.30-0.96, \mathrm{p}=0.036)$ and better $\mathrm{PFS}(\mathrm{HR}=0.1$, range: $0.03-0.33, \mathrm{p}<0.001)$. Tumor number was another independent predictor of OS (HR $=0.54$, range: $0.30-0.96, \mathrm{p}=0.036)$ and PFS $(\mathrm{HR}=0.38$, range $0.21-0.72, \mathrm{p}=0.003)$ (Table 2).

\section{Overall Survival and Progression-Free Survival Between TACE and SBRT-IO}

Figure 2 depicts the best objective response of 16 patients in SBRTIO arm and 42 evaluable patients in matched-TACE arm. Six patients of TACE arm did not have reassessment scan due to rapid deterioration. The ORR was significantly higher in the SBRT-IO group $(87.5 \%$ vs. $16.7 \%, \mathrm{p}<0.001)$. DCR was also significantly better in the SBRT-IO group $(81.3 \%$ vs. $37.5 \%, \mathrm{p}=0.002)$. In the
A

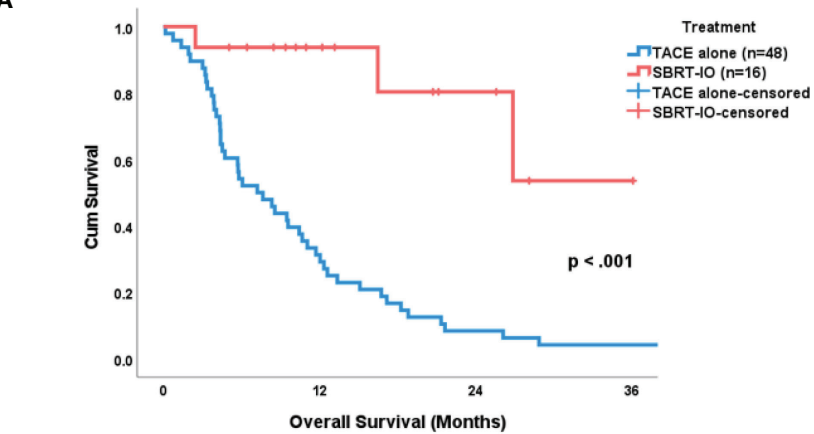

No. at risk

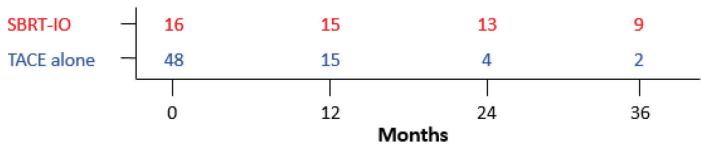

B
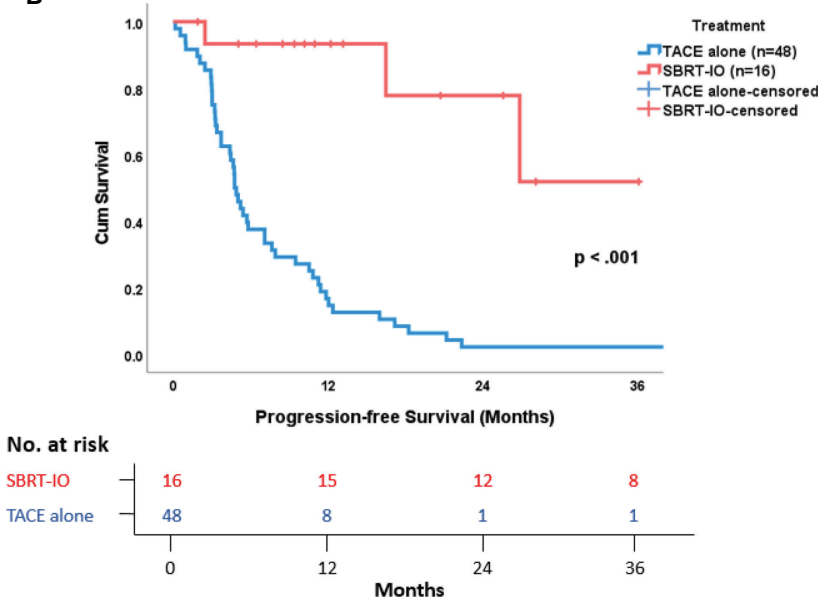

FIGURE 1 | Survival Outcome in Patients with Locally Advanced Unresectable Hepatocellular Carcinoma. (A) Overall survival and (B) Progression-free survival are remarkably and significantly better in SBRT-IO group versus matched TACE group. 
TABLE 2 | Univariate and multivariate analyses of potential prognostic factors affecting overall and progression-free survival after propensity score matching.

\begin{tabular}{|c|c|c|c|c|c|c|c|c|c|c|c|c|}
\hline \multirow[t]{3}{*}{ For matched groups $(n=64)$ : } & \multicolumn{6}{|c|}{ Overall Survival } & \multicolumn{6}{|c|}{ Progression-free Survival } \\
\hline & \multicolumn{3}{|c|}{ UVA } & \multicolumn{3}{|c|}{ MVA } & \multicolumn{3}{|c|}{ UVA } & \multicolumn{3}{|c|}{ MVA } \\
\hline & HR & $95 \% \mathrm{Cl}$ & $\mathbf{P}$ & HR & $95 \% \mathrm{Cl}$ & $\mathbf{P}$ & HR & $95 \% \mathrm{Cl}$ & $\mathbf{P}$ & HR & $95 \% \mathrm{Cl}$ & $\mathbf{P}$ \\
\hline SBRT-IO vs. TACE & 0.13 & $0.04-0.42$ & $<0.001$ & 0.14 & $0.04-0.46$ & 0.001 & 0.10 & $0.03-0.32$ & $<0.001$ & 0.10 & $0.03-0.33$ & $<0.001$ \\
\hline Age (<60 vs. $\geq 60$ years) & 1.24 & $0.58-2.67$ & 0.58 & & & & 1.37 & $0.64-2.94$ & 0.42 & & & \\
\hline Sex (male vs. female) & 1.25 & $0.49-3.16$ & 0.64 & & & & 1.50 & $0.59-3.81$ & 0.39 & & & \\
\hline Hepatitis B carrier (yes vs. no) & 1.03 & $0.59-1.81$ & 0.92 & & & & 0.99 & $0.56-1.73$ & 0.97 & & & \\
\hline ECOG (0-1 vs. 2) & 1.92 & $0.75-4.90$ & 0.17 & & & & 2.27 & $0.89-5.81$ & 0.09 & & & \\
\hline Child-Pugh class (A vs. B) & 2.08 & $0.50-8.61$ & 0.31 & & & & 1.91 & $0.46-7.92$ & 0.37 & & & \\
\hline ALBI grade (1 vs. 2 ) & 0.63 & $0.34-1.15$ & 0.13 & & & & 0.52 & $0.28-0.96$ & 0.04 & 0.90 & $0.49-1.66$ & 0.73 \\
\hline Portal vein invasion (Yes vs. no) & 1.08 & $0.55-2.12$ & 0.82 & & & & 1.13 & $0.51-2.50$ & 0.76 & & & \\
\hline BCLC stage (A vs. $B$ ) & 1.15 & $0.50-2.64$ & 0.74 & & & & 1.19 & $0.46-3.08$ & 0.72 & & & \\
\hline BCLC stage (A vs. C) & 0.77 & $0.36-1.68$ & 0.52 & & & & 0.77 & $0.31-1.87$ & 0.56 & & & \\
\hline Tumor number ( $n=1-2$ vs. $\geq 3$ ) & 0.45 & $0.25-0.80$ & 0.007 & 0.54 & $0.30-0.96$ & 0.036 & 0.38 & $0.21-0.70$ & 0.002 & 0.38 & $0.21-0.72$ & 0.003 \\
\hline Tumor size $(<10 \mathrm{~cm}$ vs. $\geq 10 \mathrm{~cm})$ & 0.60 & $0.34-1.08$ & 0.09 & & & & 0.59 & $0.33-1.05$ & 0.07 & & & \\
\hline AFP (<200 vs. $\geq 200$ ng/ml) & 0.71 & $0.40-1.25$ & 0.24 & & & & 0.67 & $0.38-1.19$ & 0.17 & & & \\
\hline
\end{tabular}

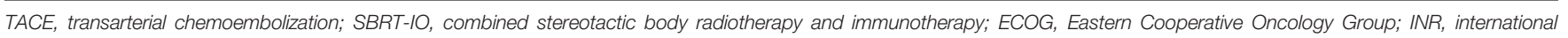
normalized ratio; BCLC, Barcelona Clinic Liver Cancer; AFP, alpha-feto protein; UVA, univariate analysis; MVA, multivariate analysis; HR, hazard ratio; Cl, confidence. interval.

matched TACE group, 24 patients (50\%) never had radiological disease controlled. The off-target progression represented the dominant mode of treatment failure in TACE arm, which occurred in 15 of 42 evaluable patients (35.7\%). In contrast, only one patient $(6.3 \%)$ developed progressive disease after SBRT-IO; this patient developed a new HCC focus outside the irradiated field and two SBRT-treated lesions had partial response (PR) and static disease (SD). Two patients of the SBRT-IO arm (12.5\%) had

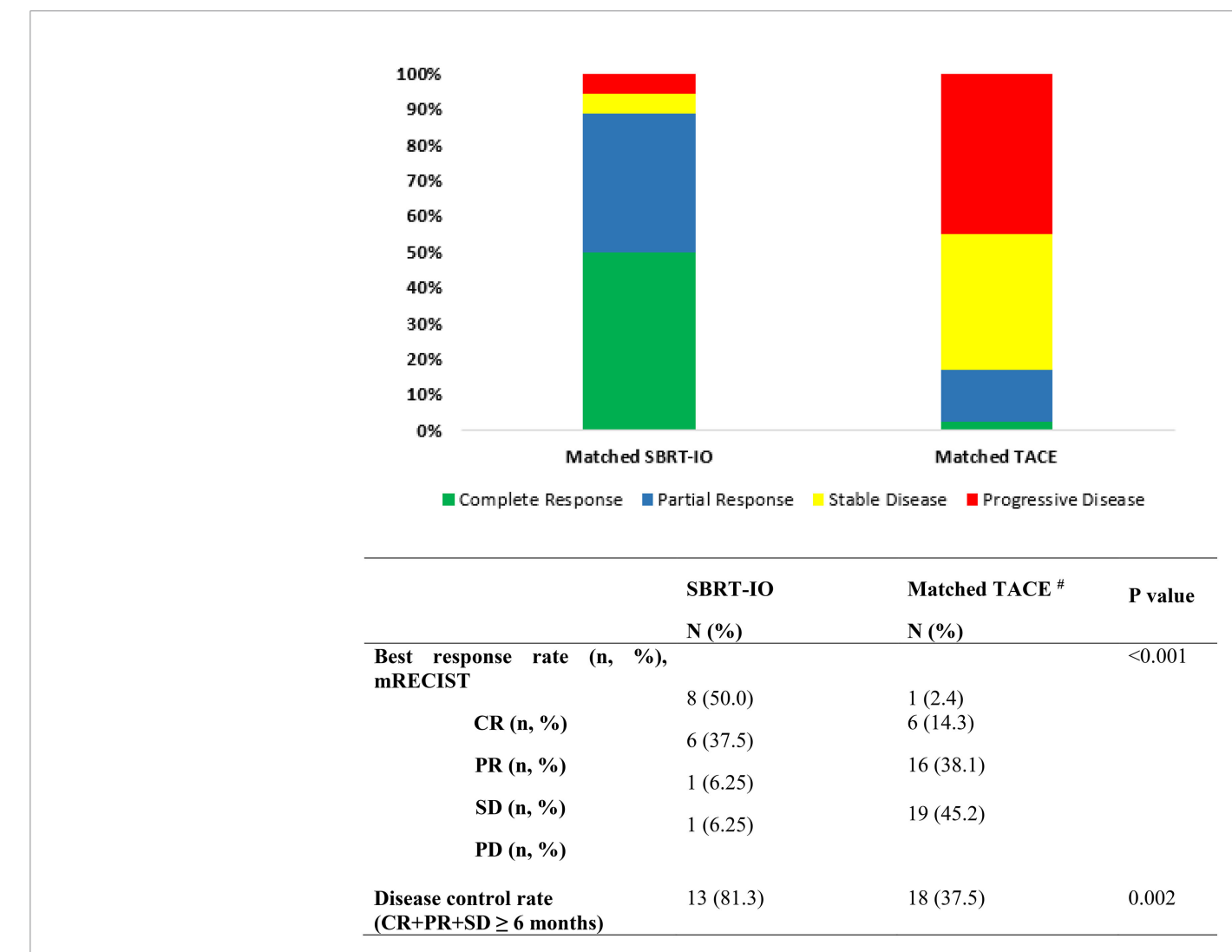

FIGURE 2 | The best mRECIST of the matched TACE and SBRT-IO patients. SBRT-IO, combined stereotactic body radiotherapy and immunotherapy; TACE, transarterial chemoembolisation; mRECIST, modified response evaluation criteria in solid tumours; CR, complete response; PR, partial response; SD, stable disease; PD, progressive disease; $\mathrm{N}$, number of lesions. \#6 subjects in the matched TACE cohort did not have follow-up scan for tumour reassessment. 
radiofrequency ablation (RFA) performed after PR, with complete clearance of the tumour subsequently achieved. The waterfall plot of Figure 3 illustrates the treatment response of index lesion in the SBRT-IO and TACE group.

Of note, Nivolumab was stopped for eight patients who achieved CR after median 7.1 months of treatment (range: 2.115.6 months); none of them developed relapse in the median follow-up time of 5.7 months (range: 0.7-25.0 months).

\section{Treatment Related Adverse Events and Liver Function Deterioration Between TACE and SBRT-IO}

Risk of $\geq$ grade 3 TRAEs and discontinuation of treatment due to toxicities were more common in patients who received TACE (60.4\% vs. $18.8 \%, \mathrm{p}=0.004 ; 25 \%$ vs. $12.5 \%$, respectively, $\mathrm{p}=$ $0.295)$. There was more elevated transaminase, anemia,
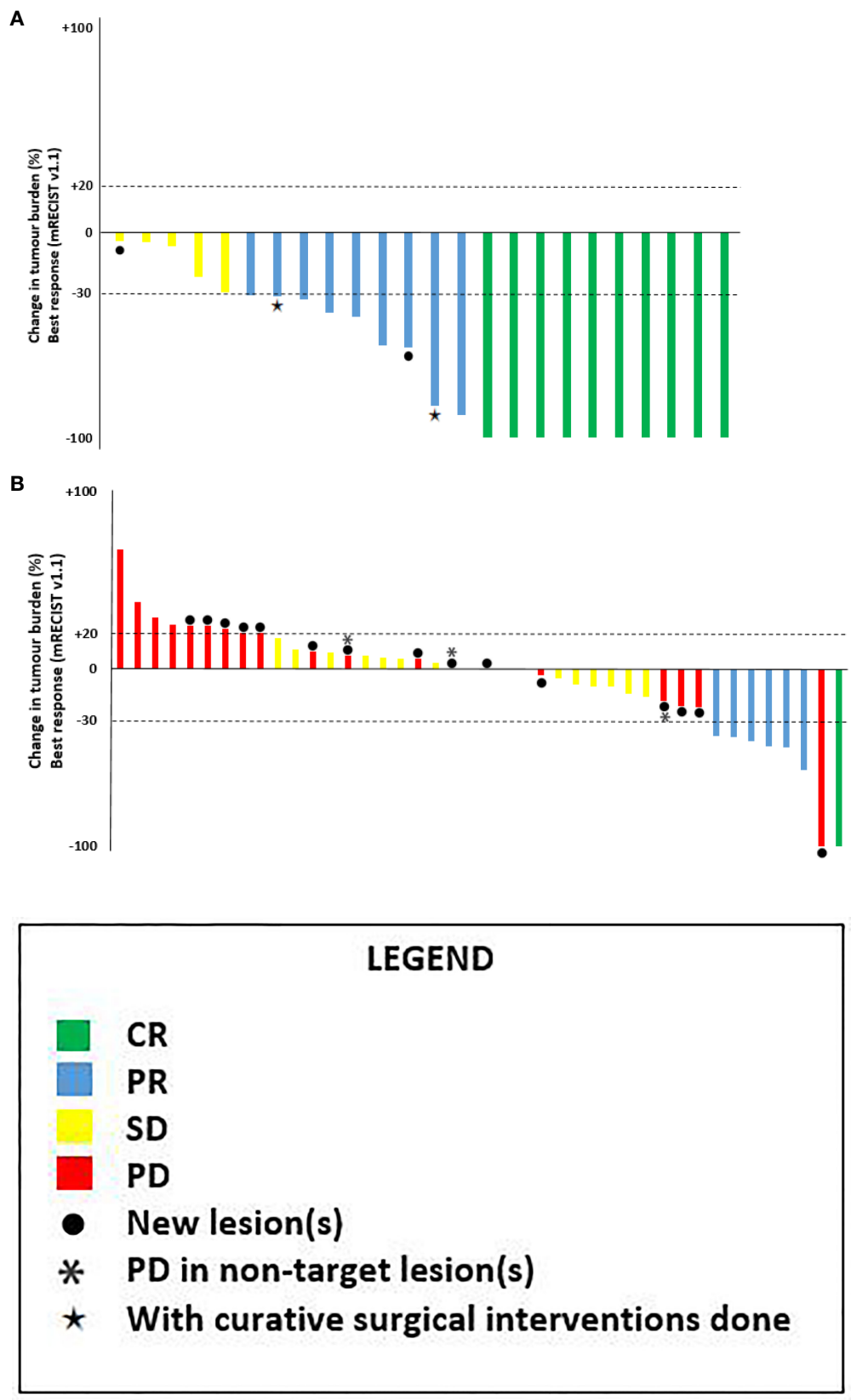

FIGURE 3 | Waterfall plots of best overall response of the target lesion(s) as per mRECIST v1.1. (A) SBRT-IO arm: 16 patients with total 24 lesions*. (B) MatchedTACE arm: 42 lesions in 48 matched patients (6 of them didn't have tumour reassessment). CR, complete response; PR, partial responses; SD, stable disease; PD, progressive disease. $\bullet$ : patients exhibiting new lesions at subsequent evaluation, ${ }^{*}$ : progression in non-target lesions, $\star$ : patients with curative surgical interventions done after responding to initial treatment. 
leukopenia, and fever in the TACE group, while patients who received SBRT-IO had more fatigue, diarrhoea, and rash. Among patients treated with SBRT-IO, none developed classical radiation induced liver disease, and there were no treatmentrelated deaths reported. There were fewer patients who developed Child-Pugh score progression $\geq 2$ at 3 months $(6.7 \%$ vs. $20.9 \%, p=0.008), 6$ months $(6.7 \%$ vs. $12.0 \%, p=0.021)$, and 12 months ( $0 \%$ vs. $21.4 \%, \mathrm{p}<0.001$ ) in the SBRT-IO arm compared to the TACE arm (Table 3).

\section{DISCUSSION/CONCLUSION}

To our knowledge, this is the first comparative study to evaluate the combined SBRT-IO in HCC population. Our findings clearly demonstrated the promising anti-tumor activity of combined SBRT-IO among patients with locally advanced HCC. This present analysis reported that patients who received SBRT-IO had statistically significant better PFS, OS, and ORR than those who received TACE. Around $90 \%$ of the patients treated with SBRT-IO survived without disease progression at 1 year and 50\% had achieved CR. The high ORR of $88.8 \%$ was also superior over that of ICI reported in previous studies (1-6).

Although TACE is widely used in patients with unresectable HCC, the prognoses vary as patients selected for TACE is highly heterogeneous in tumor burden, liver function, and treatment histories. Recent studies suggested patients with tumor burden beyond the up-to-seven criteria are unlikely to respond to TACE and their hepatic reserve tends to deteriorate after treatment (13, 14). Recently, Wang et al. showed that patients who were early refractory to TACE had significantly worse prognoses (PFS: 4.36 months vs. 12.2 months) than those responded to treatment (15). Similarly, our patient cohort had extensive tumor burden and most became refractory to TACE after median of two sessions; hence, the prognosis of matched-TACE arm was poor with median PFS 4.83 months, which is similar with the literature (15). Our study had provided a novel therapeutic approach for those who respond poorly to TACE.

There were several reasons accounted for the promising activity of SBRT-IO combination. First, previous studies had demonstrated that SBRT achieved excellent local control (1-year local control rate of 77-87\%) in locally advanced HCC, but the competing risk of metastasis had resulted in later precipitous drop of OS (median OS of 9-17 months) $(16,17)$. Notably, only one patient $(6.3 \%)$ treated with SBRT-IO in our study developed out-of-field failure compared to that of $35.7 \%$ (15 out of 42 evaluable patients) in the TACE arm. We postulated that the immune-modulatory effect of SBRT has augmented the effect of ICI in eradicating the occult metastasis; this phenomenon, known as 'systemic therapy augmented by radiotherapy

TABLE 3 | Treatment related adverse event and Child-Pugh score progression of SBRT-IO vs. matched TACE.

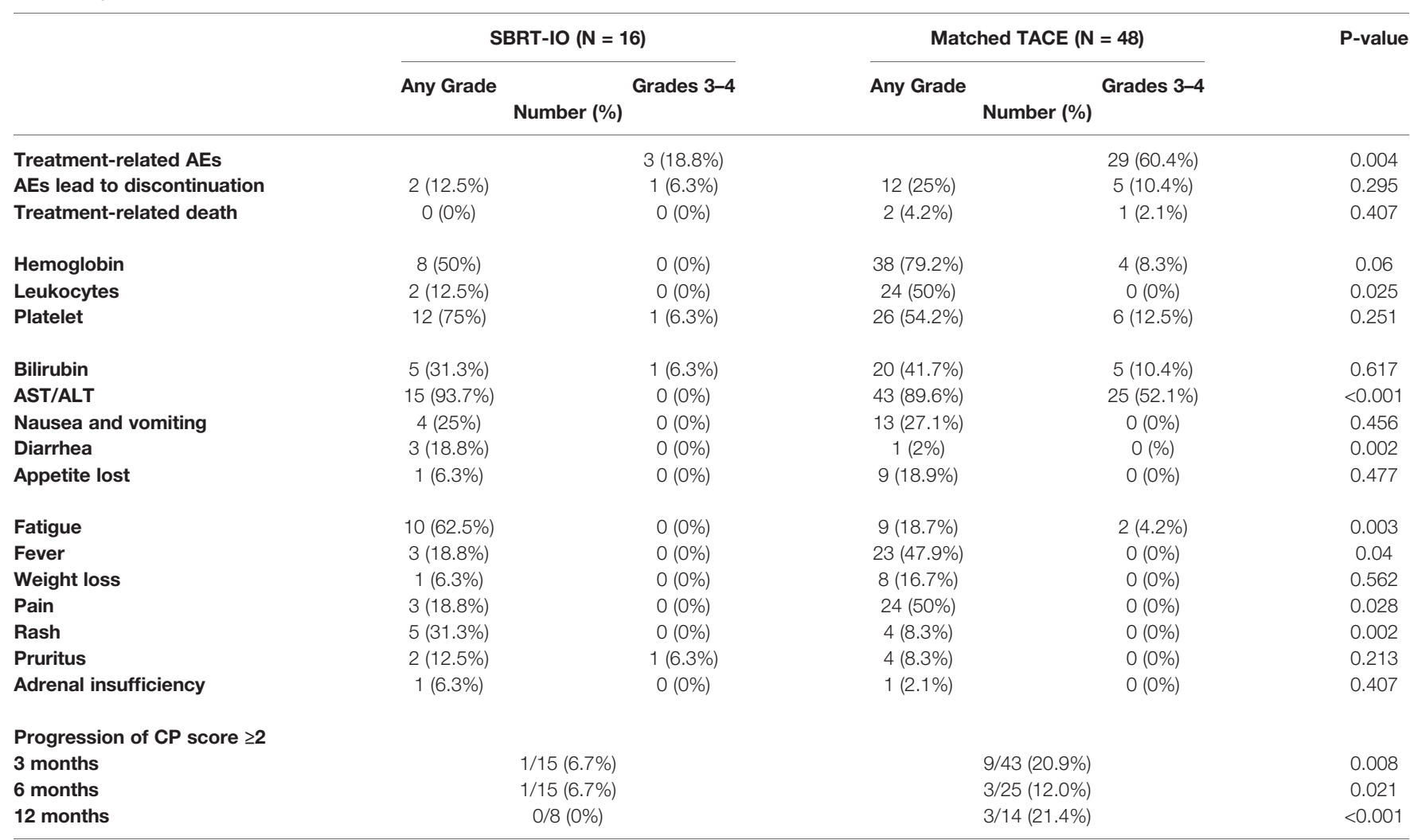

TACE, transarterial chemoembolization; SBRT-IO, combined stereotactic body radiotherapy and immunotherapy; AES, adverse events; AST, Aspartate transaminase; ALT, Alanine transaminase; $C P$, Child-Pugh.

The incidence of only toxicities $\geq 5 \%$ is shown. 
(STAR)', has also been reported in NSCLC (18). Second, because of the extensive tumor load in our patient cohort, we were only allowed to prescribe non-ablative dose of radiation (5.5-7.5 Gy $\times$ $5)$ to respect the radiation tolerance of liver $(16,17)$. Nevertheless, excellent local tumor response (ORR: 87.5\%, CR rate: $50 \%$ ) was achieved. A study by Vanpouille-Box et al. provided an important mechanistic clue regarding the modulation of the immunogenic effect by different radiation dose/fractionation schemes. They showed that modest dose radiation $(8 \mathrm{~Gy} \times 3)$ achieved similar local control as single ablative dose (30 Gy) in the concurrent use of ICI, but better systemic responses are achieved with an increased IFN- $\beta$ production via the cGAS/STING pathway. Our findings are consistent with the pre-clinical data suggesting that ICI can lower the radiation dose required to induce the same tumor response (19). Lastly, we stopped the anti-PD-1 therapy in eight patients once they attained radiological $\mathrm{CR}$; the decision was made at the discretion of clinicians in agreement with the patients. Interestingly, none of them relapsed after discontinuation of therapy for up to 2 years. The optimal duration of ICI remains unknown; yet a previous study has also suggested that the risk of progression or death is low among patients who achieved radiological CR (20). Longer follow-up is needed in our cohort to ascertain the durability of response; nevertheless, the encouraging CR rate and the durable response experienced with SBRT-IO provide hope for a cure for unresectable HCC patients without the need for additional therapy, a goal that previously seemed unachievable.

There was no abnormal safety signal observed in combined SBRT and ICI. Patients treated with SBRT-IO had better safety profile and tolerance. More importantly, treatment of TACE often leads to the deterioration of liver function that robs the patients of the opportunity of subsequent systemic therapy (13, 14). Our data suggested that SBRT-IO might better preserve the liver function of patients compared with TACE.

Our data supported the benefit of SBRT-IO in HCC patients. Prospective studies are required to validate our findings in a broader population and compare its efficacy with SBRT alone or ICI alone; future studies should also be prioritized to define the optimal timing, dosing, and treatment volume of radiotherapy and the role of PD-L1 status. Correlative studies are needed to define the mechanistic rationale behind the synergy of SBRT-IO. Lastly, recent data suggested TACE may also favorably modulate the tumor microenvironment and potentially can effectively combine with ICI; a number of trials are now on-going to evaluate the synergy of this combination (21); comparative study of SBRT-IO vs. TACE-IO is warranted when more data become available in the future.

There were several limitations of this study. First, it was a retrospective and single-center study with a small sample size; therefore, the selection bias could not be entirely eliminated. However, we had followed the patients in both arms under a unified protocol with regular imaging schedule so that the biases of PFS assessment were minimized, thus the reliability of the superior results of OS and ORR of SBRT-IO. Second, the relatively short duration of follow-up rendered the assessment of late toxicity and long-term survival not feasible. Also, the difference in the treatment period may introduce bias in favor of the survival outcome in the SBRT-IO arm, in which majority of the patients were treated in recent years with expanding therapeutic options. Nonetheless, our findings clearly demonstrated the superiority of SBRT-IO over TACE in terms of PFS and tumor response; additionally, the survival of the TACE arm has been fairly consistent over the years. Finally, the PD-L1 status and its impact on the treatment outcome were not evaluated in the present study; however, the high ORR of SBRT-IO suggested the combination worked regardless of the PD-L1 status.

In conclusion, our findings provide rationale to study the SBRT-IO treatment among locally advanced HCC patients in prospective randomized studies.

\section{DATA AVAILABILITY STATEMENT}

The original contributions presented in the study are included in the article/Supplementary Material. Further inquiries can be directed to the corresponding authors.

\section{ETHICS STATEMENT}

This study was approved by The University of Hong Kong/ Hospital Authority Hong Kong West Cluster Institutional Review Board (IRB number: UW-20-674). The patients/ participants provided their written informed consent to participate in this study. Written informed consent was not obtained from the individual(s) for the publication of any potentially identifiable images or data included in this article.

\section{AUTHOR CONTRIBUTIONS}

Conception and design: C-LC, AC, and KC. Collection and assembly of data: C-LC and AC. Data analysis and interpretation: C-LC and AC. Manuscript writing: All authors. All authors contributed to the article and approved the submitted version.

\section{ACKNOWLEDGMENTS}

We acknowledge the Li Ka Shing Foundation's 'Love can help' medical assistance program. We appreciate and acknowledge the efforts of Mr. KS Fong and Miss LY Kwan for data collection and Mr. SK Chan for statistical support.

\section{SUPPLEMENTARY MATERIAL}

The Supplementary Material for this article can be found online at: https://www.frontiersin.org/articles/10.3389/fonc.2021.798832/ full\#supplementary-material 


\section{REFERENCES}

1. El-Khoueiry AB, Sangro B, Yau T, Crocenzi TS, Kudo M, Hsu C, et al. Nivolumab in Patients With Advanced Hepatocellular Carcinoma (CheckMate 040): An Open-Label, Non-Comparative, Phase 1/2 Dose Escalation and Expansion Trial. Lancet (2017) 389:2492-502. doi: 10.1016/ S0140-6736(17)31046-2

2. Zhu AX, Finn RS, Edeline J, Cattan S, Ogasawara S, Palmer D, et al. Pembrolizumab in Patients With Advanced Hepatocellular Carcinoma Previously Treated With Sorafenib (KEYNOTE-224): A non-Randomised, Open-Label Phase 2 Trial. Lancet Oncol (2018) 19:940-52. doi: 10.1016/ S1470-2045(18)30351-6

3. Yau T, Park JW, Finn RS, Cheng A-L, Mathurin P, Edeline J, et al. CheckMate 459: A Randomized, Multi-Center Phase III Study of Nivolumab (NIVO) vs Sorafenib (SOR) as First-Line (1L) Treatment in Patients (PTS) With Advanced Hepatocellular Carcinoma (aHCC). Ann Oncol (2019) 30:v874-5. doi: 10.1093/annonc/mdz394.029

4. Finn RS, Qin S, Ikeda M, Galle PR, Ducreux M, Kim T-Y, et al. Atezolizumab Plus Bevacizumab in Unresectable Hepatocellular Carcinoma. N Engl J Med (2020) 382:1894-905. doi: 10.1056/NEJMoa1915745

5. Yau T, Kang Y-K, Kim T-Y, El-Khoueiry AB, Santoro A, Sangro B, et al. Efficacy and Safety of Nivolumab Plus Ipilimumab in Patients With Advanced Hepatocellular Carcinoma Previously Treated With Sorafenib: The CheckMate 040 Randomized Clinical Trial. JAMA Oncol (2020) 6(11): e204564. doi: 10.1001/jamaoncol.2020.4564

6. Finn RS, Ikeda M, Zhu AX, Sung MW, Baron AD, Kudo M, et al. Phase Ib Study of Lenvatinib Plus Pembrolizumab in Patients With Unresectable Hepatocellular Carcinoma. J Clin Oncol (2020) 38(26):2960-70. doi: 10.1200/JCO.20.00808

7. Bernstein MB, Krishnan S, Hodge JW, Chang JY. Immunotherapy and Stereotactic Ablative Radiotherapy (ISABR): A Curative Approach? Nat Rev Clin Oncol (2016) 13:516-24. doi: 10.1038/nrclinonc.2016.30

8. Theelen WSME, Peulen HMU, Lalezari F, van der Noort V, de Vries JF, Aerts JGJV, et al. Effect of Pembrolizumab After Stereotactic Body Radiotherapy Versus Pembrolizumab Alone on Tumor Response in Patients With Advanced Non-Small Cell Lung Cancer: Results of the PEMBRO-RT Phase 2 Randomized Clinical Trial. JAMA Oncol (2019) 5:1276-82. doi: 10.1001/ jamaoncol.2019.1478

9. Bauml JM, Mick R, Ciunci C, Aggarwal C, Davis C, Evans T, et al. Pembrolizumab After Completion of Locally Ablative Therapy for Oligometastatic Non-Small Cell Lung Cancer: A Phase 2 Trial. JAMA Oncol (2019) 5:1283-90. doi: 10.1001/jamaoncol.2019.1449

10. Chiang CL, Chan ACY, Chiu KWH, Kong F-MS. Combined Stereotactic Body Radiotherapy and Checkpoint Inhibitor in Unresectable Hepatocellular Carcinoma: A Synergistic Treatment Strategy. Front Oncol (2019) 9:1157. doi: 10.3389/fonc.2019.01157

11. Sharabi AB, Lim M, DeWeese TL, Drake CG. Radiation and Checkpoint Blockade Immunotherapy: Radiosensitisation and Potential Mechanisms of Synergy. Lancet Oncol (2015) 16(13):e498-509. doi: 10.1016/S1470-2045(15)00007-8

12. Lo C, Ngan H, Tso W, Liu C, Lam C-M, Poon RT-P, et al. Randomized Controlled Trial of Transarterial Lipiodol Chemoembolization for Unresectable Hepatocellular Carcinoma. Hepatology (2002) 35(5):1164-71. doi: 10.1053/jhep.2002.33156

13. Yasui Y, Tsuchiya K, Kurosaki M, Takeguchi T, Takeguchi Y, Okada M, et al. Up-To-Seven Criteria as a Useful Predictor for Tumor Downstaging to
Within Milan Criteria and Child-Pugh Grade Deterioration After Initial Conventional Transarterial Chemoembolization. Hepatol Res (2018) 48:442-50. doi: 10.1111/hepr.13048

14. Arizumi T, Minami T, Chishina H, Kono M, Takita M, Yada N, et al. Time to Transcatheter Arterial Chemoembolization Refractoriness in Patients With Hepatocellular Carcinoma in Kinki Criteria Stages B1 and B2. Dig Dis (2017) 35:589-97. doi: 10.1159/000480208

15. Wang TC, An TZ, Li JX, Zhang Z-S, Xiao Y-D. Development and Validation of a Predictive Model for Early Refractoriness of Transarterial Chemoembolization in Patients With Hepatocellular Carcinoma. Front Mol Biosci (2021) 8:633590. doi: 10.3389/fmolb.2021.633590

16. Bujold A, Massey C, Kim J, Brierley J, Cho C, Wong RKS, et al. Sequential Phase I and II Trials of Stereotactic Body Radiotherapy for Locally Advanced Hepatocellular Carcinoma. J Clin Oncol (2013) 31:1631-9. doi: 10.1200/ JCO.2012.44.1659

17. Gkika E, Schultheiss M, Bettinger D, Maruschke L, Neeff HP, Schulenburg M, et al. Excellent Local Control and Tolerance Profile After Stereotactic Body Radiotherapy of Advanced Hepatocellular Carcinoma. Radiat Oncol (2017) 12:116. doi: 10.1186/s13014-017-0851-7

18. Torok JA, Samama JK. Combining Immunotherapy and Radiotherapy for the STAR Treatment. Nat Rev Clin Oncol (2019) 16:666-7. doi: 10.1038/s41571019-0277-2

19. Vanpouille-Box C, Alard A, Aryankalayil MJ, Sarfraz Y, Diamond JM, Schneider RJ, et al. DNA Exonuclease Trex1 Regulates RadiotherapyInduced Tumour Immunogenicity. Nat Commun (2017) 8:15618. doi: 10.1038/ncomms 15618

20. Jansen YJL, Rozeman EA, Mason R, Goldinger SM, Geukes Foppen MH, Hoejberg L, et al. Discontinuation of Anti-PD-1 Antibody Therapy in the Absence of Disease Progression or Treatment Limiting Toxicity: Clinical Outcomes in Advanced Melanoma. Ann Oncol (2019) 30:1154-61. doi: 10.1093/annonc/mdz110

21. Greten TF, Mauda-Havakuk M, Heinrich B, Korangy F, Wood BJ. Combined Locoregional-Immunotherapy for Liver Cancer. J Hepatol (2019) 70(5):9991007. doi: 10.1016/j.jhep.2019.01.027

Conflict of Interest: C-LC reports receiving research funding of AstraZeneca, Merck Kgga, and Taiho. He had a consulting or advisory role at AstraZeneca and Eiasi.

The remaining authors declare that the research was conducted in the absence of any commercial or financial relationships that could be construed as a potential conflict of interest.

Publisher's Note: All claims expressed in this article are solely those of the authors and do not necessarily represent those of their affiliated organizations, or those of the publisher, the editors and the reviewers. Any product that may be evaluated in this article, or claim that may be made by its manufacturer, is not guaranteed or endorsed by the publisher.

Copyright (๑) 2021 Chiang, Chiu, Lee, Kong and Chan. This is an open-access article distributed under the terms of the Creative Commons Attribution License (CC BY). The use, distribution or reproduction in other forums is permitted, provided the original author(s) and the copyright owner(s) are credited and that the original publication in this journal is cited, in accordance with accepted academic practice. No use, distribution or reproduction is permitted which does not comply with these terms. 